\title{
Least-Squares Collocation Bernstein Method for Solving System of Linear Fractional Integro-diffrential Equations
}

\author{
Oyedepo Taiye \\ Federal College of Dental Technology \\ and Therapy, Enugu, Nigeria
}

\author{
Ayinde Muhammed Abdullahi \\ Modibbo Adama University, Yola, \\ Nigeria
}

\author{
Adenipekun Adewale \\ Emmanuel \\ Federal Polytechnic Ede, Osun, \\ Nigeria
}

\author{
Ajileye Ganiyu \\ Federal University Wukari, Taraba, Nigeria
}

\begin{abstract}
This study gears towards finding a new simple numerical algorithm to solve system of linear fractional integrodifferential equations. The technique involves the application of Caputo properties, the properties of Bernstein polynomials and least square collocation approach to reduce the problem to system of linear algebraic equations and then solved. To demonstrate the accuracy and applicability of the presented method some numerical examples are given. Numerical results show that the method is easy to implement and compares favorably with the exact results. The graphical solution of the method is displayed.
\end{abstract}

\section{Keywords}

System of linear fractional integro- differential equations; least squares collocation; Bernstein polynomials

\section{INTRODUCTION}

Fractional integro-differential equations has played a significant role in modelling of real world physical problems e.g the modeling of earthquake, reducing the spread of virus, control the memory behaviour of electric socket and many others. Fractional calculus is a field dealing with integral and derivatives of arbitrary orders, and their applications in science, engineering and other fields. The idea is from the ordinary calculus. According to [1-3], It was discovered by Leibniz in the year 1695 few years after he discovered ordinary calculus but later forgotten due to the complexity of the formula. Since most Fractional Integro-diffrential Equations (FIDEs) cannot be solved analytically, much attention has been devoted to search for approximate and numerical techniques to the solution of FIDEs. Recently, many methods have been developed by researchers for providing approximate solutions of FIDEs. [4] employed Lagurre polynomials as basis functions for the solution of fractional Solving Fredholm integro-differential equations while [5] employed Bernstein polynomials as basis functions to approximate the solution of FIDEs. References [6 - 8] applied collocation techniques for solving FIDEs using different basis functions. [9] applied Sumudu transform method and Hermite Spectral collocation method for solving FIDEs. Author [10] introduced approximate solutions of Volterra-Fredholm integro-differential equations of fractional order. References [11 - 12] used Least - Squares method for the solution of FIDEs. [13 - 15] introduced numerical solution of fractional singular integro-differential equations by using Taylor series expansion and Galerkin method and a fast numerical algorithm based on the second kind of Chebyshev polynomials. The author in [16] applied numerical solution of Fredholm-Volterra fractional integro-differential equation with nonlocal boundary conditions. Reference [17] employed Bernstein modified homotopy perturbation method for the Solution of Volterra fractional integro-differential equations. The objective of this work is to introduce a new technique called least squares collocation Bernstein method with application of Caputo properties that provide less rigorous works in terms of computational cost with improved accuracy for finding an approximate solution to system of Linear fractional integro- differential equations. The general form of the class of problem considered in this work is given as:

$D^{\alpha} u_{i}(x)=p_{i}(x) u_{i}(x)+f_{i}(x)+\int_{0}^{x} k_{i}(x, t)\left(\sum_{r=1}^{n} u_{r}(t)\right) d t$ $i=1,2, \ldots, n, o \leq x, t \leq 1$,

With the following supplementary conditions:

$u_{i}{ }^{(j)}\left(x_{0}\right)=u_{i j} i=0,1,2, \ldots, m-1$,

$m-1<\alpha \leq m, m \in, N$

Where $D^{\alpha} u_{i}(x)$ indicates the $\propto$ th Caputo fractional derivative of $u_{i}(x) ; p_{i}(x), f_{i}(x), k_{i}(x, t)$ are given smooth functions, $x$ and $t$ are real variables varying $[0,1]$ and $u_{i}(x)$ is the unknown function to be determined.

\section{SOME RELEVANT BASIC DEFINITIONS}

Definition 2.1: Riemann - Lowville fractional integral is defined as [18]:

$$
\begin{aligned}
& J^{\alpha} f(x)=\frac{1}{\Gamma(\alpha)} \int_{0}^{x} \frac{f(x)}{(x-t)^{1-\alpha}} d t, \alpha>0, x>0, \\
& J^{\alpha} \text { denotes the fractional integral of order } \propto
\end{aligned}
$$

Definition 2.2: The Caputo Factional Derivative is defined as [18]:

$D^{\alpha} f(x)=\frac{1}{\Gamma(\mathrm{n}-\alpha)} \int_{0}^{x}(x-s)^{n-\alpha-1} f^{m}(s) d s$

Where $m$ is a positive integer with the property that $n-1<$ $\propto<n$

For example if $0<\propto<1$ the caputo fractional derivative is

$D^{\alpha} f(x)=\frac{1}{\Gamma(1-\alpha)} \int_{0}^{x}(x-s)^{-\alpha} f^{1}(s) d s$

Hence, we have the following properties:

(1) $J^{\alpha} J^{v} f=j^{\alpha+v} f, \alpha, v>0, f \in C_{\mu}, \mu>0$

(2) $J^{\alpha} x^{\gamma}=\frac{\Gamma(\lambda+1)}{\Gamma(\alpha+\gamma+1)} x^{\alpha+\gamma}, \alpha>0, \gamma>-1, x>0$

(3) $J^{\alpha} \quad D^{\alpha} f(x)=f(x)-\sum_{k=0}^{n-1} f^{k}(0) \frac{x^{k}}{k !}, \quad x>$ $0, n-1<\alpha \leq n$

(4) $D^{\alpha} J^{\alpha} f(x)=f(x), \quad x>0, n-1<\alpha \leq n$,

(5) $D^{\alpha} C=0, C$ is the constant, 


$$
\text { (6) }\left\{\begin{array}{cc}
0, & \beta \in N_{0}, \beta<[\alpha], \\
D^{\alpha} x^{\beta}=\frac{\Gamma(\beta+1)}{\Gamma(\beta-\alpha+1)} x^{\beta-\alpha}, & \beta \in N_{0}, \beta \geq[\alpha],
\end{array}\right.
$$

Where $[\alpha]$ denoted the smallest integer greater than or equal to $\alpha$ and $N_{0}=\{0,1.2, \ldots\}$

Definition 2.3: Bernstein basis polynomials: A Bernstein polynomial [19] of degree $N$ is defined by

$$
B_{i, m}(x)=\left(\begin{array}{c}
m \\
i
\end{array}\right) x^{i}(1-x)^{m-i} \quad i=0,1 \ldots n,
$$

where,

$$
\left(\begin{array}{c}
m \\
i
\end{array}\right)=\frac{m !}{i !(m-1) !}
$$

Often, for mathematical convenience, we set $B_{i, m}(x)=0$ if $<0$ or $j>m$

Definition 2.4: Bernstein polynomials: A linear combination Bernstein [19] basis polynomials $u_{i}(x)=\sum_{j}^{m} a_{j}{ }^{i} u_{j}(x)$

the Bernstein polynomial of degree $\mathrm{n}$ where $a_{j}, j=$ $0,1,2, \ldots \ldots .$. are constants.

\section{DEMONSTRATION OF LEAST SQUARES COLLOCATION BERNSTEIN METHOD (LSCBM)}

The Least Squares Collocation Bernstein Method is based on approximating the unknown function $u_{i}(x)$ in (1) by assuming an approximate solution of the form defined in (8). Consider equation (1) operating with $J^{\propto}$ on both sides as follows:

$$
\begin{aligned}
& J^{\alpha}\left[u_{i}(x)\right]= \\
& J^{\alpha}\left[p_{i}(x) u_{i}(x)+f_{i}(x)+\int_{0}^{x} k_{i}(x, t)\left(\sum_{v=1}^{n} u_{v}(t)\right) d t\right] \\
& u_{i}(x)=\sum_{k=0}^{n-1} u_{i}^{k}(0) \frac{x^{k}}{k !}+J^{\alpha}\left[p_{i}(x) u_{i}(x)+f_{i}(x)+\right. \\
& \left.\int_{0}^{x} k_{i}(x, t)\left(\sum_{r=1}^{n} u_{r}(t)\right) d t\right] \\
& \text { Substituting (8) into (10) }
\end{aligned}
$$$$
\sum_{j=0}^{m} a_{j}{ }^{i} u_{j}(x)=\sum_{k=0}^{m-1} u_{i}^{k}(0) \frac{x^{k}}{k !}+J^{\alpha}\left[p_{i}(x) \sum_{j}^{m} a_{j}{ }^{i} u_{j}(x)+\right.
$$$$
\left.f_{i}(x)+\int_{0}^{x} k_{i}(x, t)\left(\sum_{r=1}^{n}\left[\sum_{j=0}^{m} a_{j}^{i} u_{j}(t)\right]\right) d t\right]
$$

Hence, the residual equation is obtained as

$$
R\left(a_{0}{ }^{i}, a_{1}{ }^{i}, \ldots, a_{m}{ }^{i}\right)=
$$$$
\sum_{j=0}^{m} a_{j}{ }^{i} u_{j}(x)-\left\{\sum_{k=0}^{m-1} u_{i}^{k}(0) \frac{x^{k}}{k !}+\right.
$$$$
J^{\alpha}\left[p_{i}(x) \sum_{j=0}^{m} a_{j}{ }^{i} u_{j}(x)+f_{i}(x)+\right.
$$$$
\left.\left.\int_{0}^{x} k_{i}(x, t)\left(\sum_{r=1}^{n}\left[\sum_{j=0}^{m} a_{j}^{i} u_{j}(t)\right]\right) d t\right]\right\}
$$

Let

$$
S\left(a_{0}, a_{1} \ldots, a_{m}\right)=\left[R\left(a_{0}, a_{1}, \ldots \ldots, a_{m}\right)\right]^{2} w(x)
$$

Where $w(x)$ is the positive weight function defined in the interval, $[\mathrm{a}, \mathrm{b}]$. In this work,

we take $w(x)=1$ for simplicity. Thus,

$S\left(a_{0}{ }^{i}, a_{1}{ }^{i}, \ldots, a_{m}{ }^{i}\right)=\left\{\sum_{j=0}^{m} a_{j}{ }^{i} u_{j}(x)-\left\{\sum_{k=0}^{m-1} u_{i}{ }^{k}(0) \frac{x^{k}}{k !}+\right.\right.$ $J^{\propto}\left[p(x) \sum_{j=0}^{m} a_{j}^{i} u_{j}(x)+f_{i}(x)+\right.$

$\left.\left.\left.\int_{0}^{x} k_{i}(x, t)\left(\sum_{r=1}^{n}\left[\sum_{j=0}^{m} a_{j}^{i} u_{j}(t)\right]\right) d t\right]\right\}\right\}^{2}$

In order to minimize equation (15), we obtained the values of $a_{j}(j \geq 0)$ by finding the minimum value of $S$ as:

$$
\frac{\partial S_{i}}{\partial a_{j}{ }^{i}}=0, j=0,1,2 \ldots, m
$$

Applying (15) on (14), we have

$\left\{\sum_{j=0}^{m} a_{j}^{i} u_{j}(x)-\left\{\sum_{k=0}^{m-1} u_{i}^{k}(0) \frac{x^{k}}{k !}+\right.\right.$

$J^{\propto}\left[p(x) \sum_{j=0}^{m} a_{j}^{i} u_{j}(x)+f_{i}(x)+\right.$

$\left.J^{\propto}\left[\int_{0}^{x} k_{i}(x, t)\left(\sum_{r=1}^{n}\left[\sum_{j=0}^{m} a_{j}{ }^{i} u_{j}(t)\right]\right) d t\right]\right\} \times\left\{u_{j}(x)-\right.$

$\left.J^{\propto}\left[p(x) u_{j}(x)\right]-J^{\propto}\left[\int_{0}^{x} k_{i}(x, t)\left(\sum_{r=1}^{n}\left[\sum_{j=0}^{m} u_{j}(t)\right]\right) d t\right]\right\}$
Thus, (16) is then simplified for $j=0,1, \ldots n$ and collocated at equally spaced point $x_{i}=a+\frac{(b-a) i}{m},(i=1(1) m)$ to obtain $(m+1)$ algebraic system of equations in $(m+1)$ unknown $a_{j}^{i}$ which are then put in matrix form as follow:

$A=$

$\left(\begin{array}{cccc}R_{i}\left(x, a_{0}{ }^{i}\right) h_{0}{ }^{i} & R_{i}\left(x, a_{1}{ }^{i}\right) h_{0}{ }^{i} \cdots & R_{i}\left(x, a_{m}{ }^{i}\right) h_{0}{ }^{i} \\ R_{i}\left(x, a_{0}{ }^{i}\right) h_{1}{ }^{i} & R_{i}\left(x, a_{1}{ }^{i}\right) h_{1}{ }^{i} \ldots & R_{i}\left(x, a_{m}{ }^{i}\right) h_{1}{ }^{i} \\ \vdots & \vdots & \ddots & \vdots \\ R_{i}\left(x, a_{m}{ }^{i}\right) h_{m}{ }^{i} & R_{i}\left(x, a_{m}{ }^{i}\right) h_{m}{ }^{i} & \ldots & R_{i}\left(x, a_{j}{ }^{i}\right) h_{m}{ }^{i}\end{array}\right)$,

$$
B=\left(\begin{array}{c}
{\left[J^{\propto} f_{i}(x)+\sum_{k=0}^{n-1} u_{i}{ }^{k}(0) \frac{x^{k}}{k !}\right] h_{0}{ }^{i}} \\
{\left[J^{\propto} f_{i}(x)+\sum_{k=0}^{n-1} u_{i}{ }^{k}(0) \frac{x^{k}}{k !}\right] h_{1}{ }^{i}} \\
\vdots \\
{\left[J^{\propto} f_{i}(x)+\sum_{k=0}^{n-1} u_{i}{ }^{k}(0) \frac{x^{k}}{k !}\right] h_{m}{ }^{i}}
\end{array}\right)
$$

Where

$$
\begin{gathered}
h_{j}{ }^{i}= \\
u_{j}(x)-J^{\propto}\left[p(x) u_{j}(x)\right]- \\
J^{\propto}\left[\int_{0}^{x} k_{i}(x, t)\left(\sum_{r=1}^{n}\left[\sum_{j=0}^{m} u_{j}(t)\right]\right) d t\right], \quad j=0,1, \ldots, m \\
R_{i}\left(x, a_{j}^{i}\right)= \\
\sum_{j=0}^{m} a_{j}{ }^{i} u_{j}(x)-\left\{J ^ { \alpha } \left[p(x) \sum_{j=0}^{m} a_{j}{ }^{i} u_{j}(x)+\right.\right. \\
J^{\alpha}\left[\int_{0}^{x} k_{i}(x, t)\left(\sum_{r=1}^{n}\left[\sum_{j=0}^{m} a_{j}{ }^{i} u_{j}(t)\right]\right) d t\right], \\
\quad j=0,1, \ldots, m, \quad I=1,2, \ldots, n
\end{gathered}
$$

The $(m+1)$ linear equations are then solved to obtain the unknown constants $a_{j}(j=0(1) m)$, which are then substituted back into the assumed approximate solution to give the required approximation solution.

\section{NUMERICAL EXAMPLES}

In this section, the above technique is implemented on some problems. The problems are then solved via the Bernstein polynomials as basis functions. The problems are then solved to illustrate the computational cost accuracy and efficiency of the proposed method using Maple 18.

Example 5.1: Consider the following fractional Integrodifferential [20]

$$
\begin{aligned}
& D^{\propto} u_{1}(x)=-\frac{x}{6}+\frac{3 x^{\frac{1}{3}}}{\Gamma\left(\frac{1}{3}\right)}+\int_{0}^{1} 2 x t\left[u_{1}(t)+u_{2}(t)\right] d t, \\
& D^{\frac{2}{3}} u_{2}(x)=-\frac{5 x^{3}}{6}+\frac{9 x^{\frac{4}{3}}}{2 \Gamma\left(\frac{1}{3}\right)}+\int_{0}^{1} x^{3}\left[u_{1}(t)-u_{2}(t)\right] d t
\end{aligned}
$$

Subject to initial condition $u_{1}(0)=-1, u_{2}(0)=0$ with the exact solution $u_{1}(x)=x-1, u_{2}(x)=x^{2}$

Applying above mothed on example 1 , taking $\propto=\frac{3}{4}$ and $m=2$. The following constants were obtained as: $a_{0}=$ $-1.000000014, a_{1}=-0.4999999007, a_{2}=0$

for equation (21) and $a_{0}=9.696724573 \times 10^{-10}, a_{1}=$ $-4.132001054 \times 10^{-9} 0, a_{2}=1.000000011$ for equation (22). Substituting the values back into the assumed approximate solution, we obtain the approximate solution as: $u_{1}(x)=-2.126 \times 10^{-7} x^{2}+1.0000000227 x-$ 1.000000014 , $u_{2}(x)=1.000000020 x^{2}-1.020334702 \times 10^{-8} x+$ $9.696724573 \times 10^{-10}$. Comparing the result obtained by [20] with the new method, it tends to be said that the proposed method performed more accurately since the table of error found is smaller than [20] and the graph of the approximate solution is the same as the graph of exact solution. 


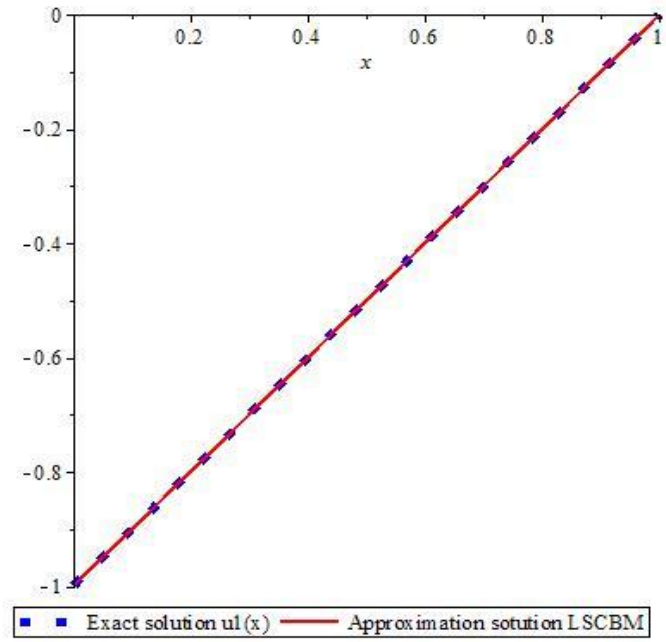

Figure 1: Showing the graph of approximation solution $u_{1}(x)$ and exact of example 1

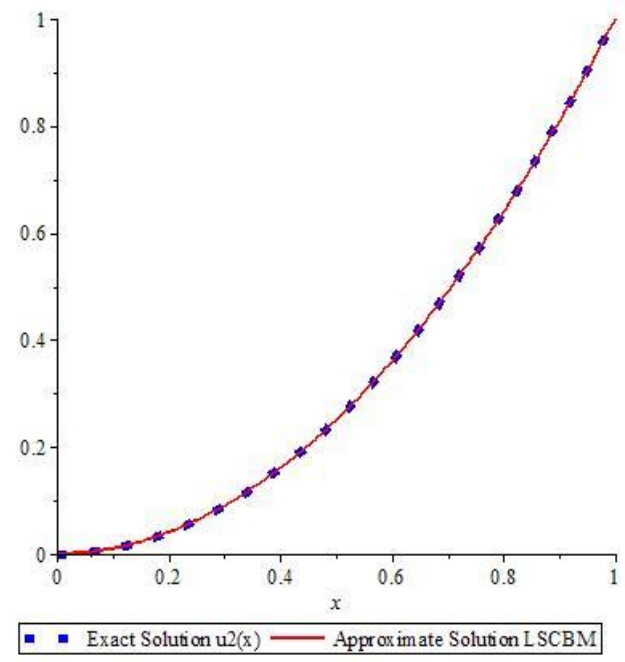

Figure 2: Showing the graph of approximation solution $u_{2}(x)$ and exact of example 1

Example 5.2: Consider the following fractional Integrodifferential [20]

$D^{\propto} u_{1}(x)=-\frac{1}{20}-\frac{x}{12}+\frac{4 x^{\frac{1}{4}}\left(15-23 x^{2}\right)}{15 \Gamma\left(\frac{1}{3}\right)}+\int_{0}^{1}(x+t)\left[u_{1}(t)+\right.$

$\left.u_{2}(t)\right] d t$

$D^{\propto} u_{2}(x)=-\frac{5 x^{3}}{6}+\frac{9 x^{\frac{4}{3}}}{2 \Gamma\left(\frac{1}{3}\right)}+\int_{0}^{1} x^{\frac{1}{2}} t^{2}\left[u_{1}(t)-u_{2}(t)\right] d t$

Subject to initial condition $u_{1}(0)=0, u_{2}(0)=0$ with the exact solution $u_{1}(x)=x-x^{3}, u_{2}(x)=x^{2}-x$.

Solving Example 2, following the same procedure above, we take $\propto=\frac{3}{4}$ and $m=3$. The following constants were obtained as: $a_{0}=9.030799779 \times 10^{-9}, a_{1}=0.3333335125$ , $a_{2}=0.6666672478, a_{3}=0$ for equation (23) and $a_{0}=$ $0, a_{1}=-0.3333333432, a_{2}=-0.3333333621, a_{3}=0$ for equation (24). Substituting the values back into the assumed approximate solution, the approximate solution is obtained as:

$u_{1}(x)=-1.000001214 x^{3}+6.95 \times 10^{-7} x^{2}+$

$1.000000511 x+9.0307997$

$u_{2}(x)=5.6 \times 10^{-10} x^{3}+0.9999999 x^{2}-1.000000030 t$.

.Comparing the result obtained by [20] with the new method, it tends to be said that the proposed method performed more accurately since the table of error found is smaller than [20] and the graph of the approximate solution is the same as the graph of exact solution

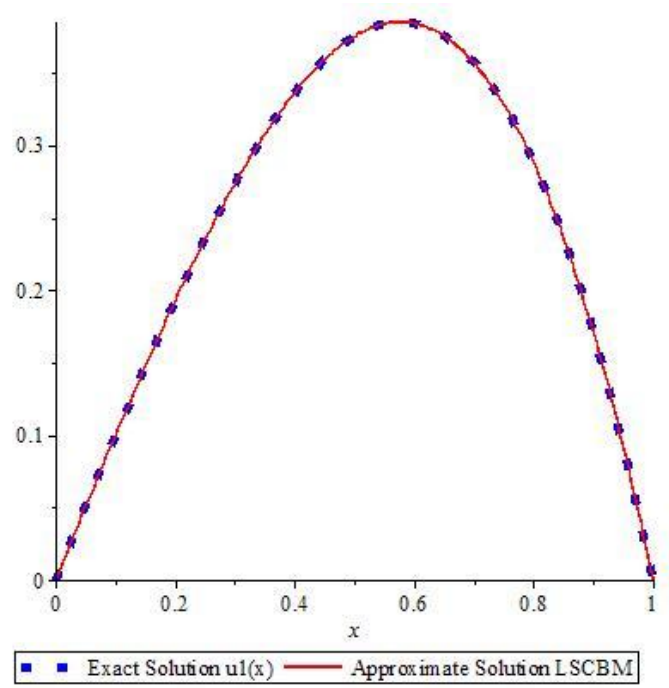

Figure 3: Showing the graph of approximation solution $u_{1}(x)$ and exact of example 2

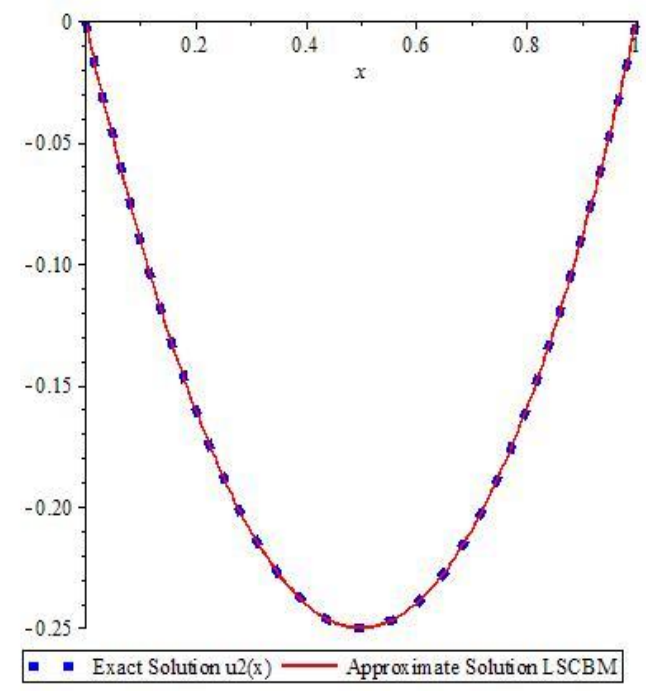

Figure 4: Showing the graph of approximation solution $u_{2}(x)$ and exact of example 2

Example 5.3: Consider the following fractional Integrodifferential [20]

$D^{\frac{4}{5}} u_{1}(x)=\frac{83 x}{80}+\frac{x}{12}+\frac{25 x^{\frac{6}{5}}(11+15 x)}{33 \Gamma\left(\frac{1}{5}\right)}+\int_{0}^{1} 2 x t\left[u_{1}(t)+\right.$

$\left.u_{2}(t)\right] d t$

$D^{\frac{3}{4}} u_{2}(x)=-\frac{5 x^{3}}{6}+\frac{9 x^{\frac{4}{3}}}{2 \Gamma\left(\frac{1}{3}\right)}+\int_{0}^{1}(x+t)\left[u_{1}(t)-u_{2}(t)\right] d t$.

Subject to initial condition $u_{1}(0)=0, u_{2}(0)=0$ with the exact solution $u_{1}(x)=x^{3}-x^{2}, u_{2}(x)=\frac{15}{8} x^{2}$. Similarly solving Example 3, following the same procedure above, we take $\propto=\frac{3}{4}$ and $m=2$. The following constants were obtained as: $a_{0}=0, a_{1}=0, a_{2}=0.3333333306, a_{3}=0$ for equation (25) and $a_{0}=0, a_{1}=0, a_{2}=0.6249996843, a_{3}=$ 1.8749993560 for equation (26). Substituting the values back 
into the assumed approximate solution, the approximate solution is obtained as:

$u_{1}(x)=0.9999999918 x^{3}-0.9999999918 x^{3}$,

$u_{2}(x)=3.03 \times 10^{-7} x^{3}+1.87499053 x^{2}$.Comparing the result obtained by [20] with the new method, it tends to be said that the proposed method performed more accurately since the table of error found is smaller than [20] and the graph of the approximate solution is the same as the graph of exact solution

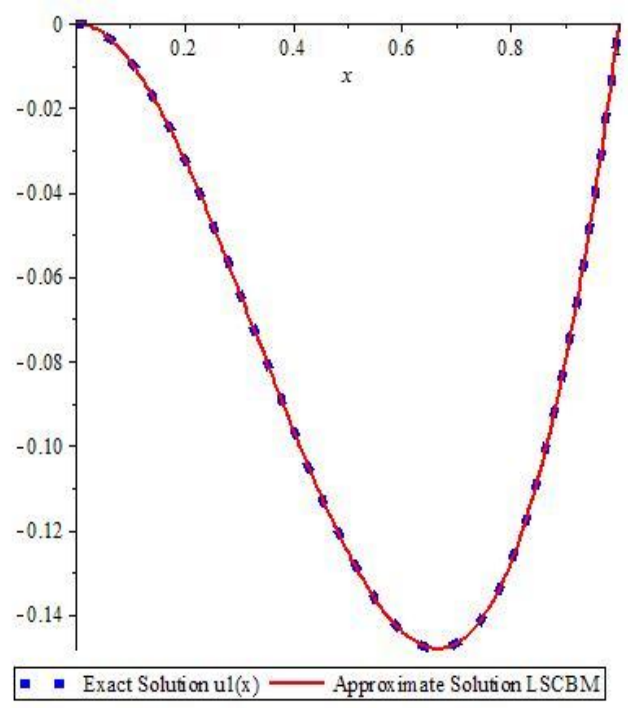

Figure 5: Showing the graph of approximation solution $u_{1}(x)$ and exact of example 3

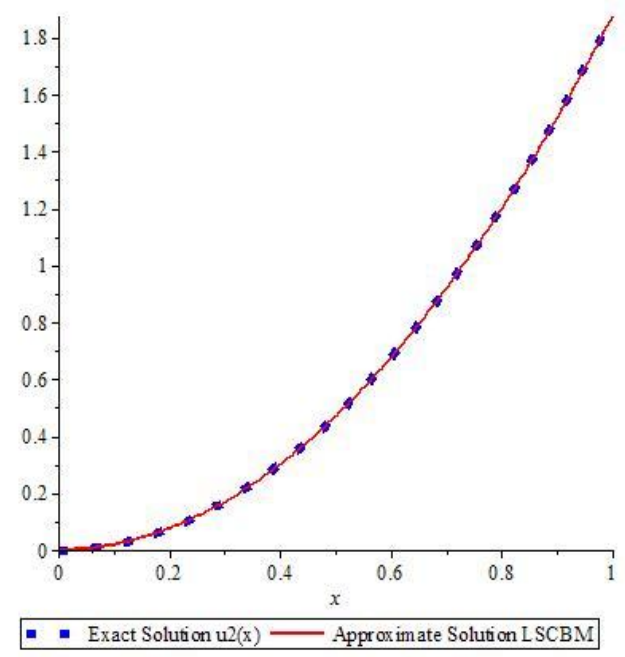

Figure 6: Showing the graph of approximation solution $u_{2}(x)$ and exact of example 3

\section{TABLE OF RESULTS}

Table 1. Comparison of the absolute errors for Example 1

\begin{tabular}{|l|c|c|}
\hline $\mathrm{X}$ & $\mathrm{LSCBM} u_{1}(\boldsymbol{x})$ & $\mathrm{ADM}[20]$ \\
\hline 0.0 & $1.400 \times 10^{-8}$ & $7.776 \times 10^{-5}$ \\
\hline 0.2 & $2.290 \times 10^{-8}$ & $2.468 \times 10^{-4}$ \\
\hline 0.4 & $4.278 \times 10^{-8}$ & $7.838 \times 10^{-4}$ \\
\hline 0.6 & $4.566 \times 10^{-8}$ & $1.540 \times 10^{-3}$ \\
\hline 0.8 & $3.154 \times 10^{-8}$ & $2.488 \times 10^{-3}$ \\
\hline 1.0 & $4.000 \times 10^{-8}$ & $3.609 \times 10^{-3}$ \\
\hline
\end{tabular}

Table 2. Comparison of the absolute errors for Example 1

\begin{tabular}{|l|c|c|}
\hline $\mathrm{X}$ & $\mathrm{LSCBM} u_{2}(\boldsymbol{x})$ & $\mathrm{ADM}[20]$ \\
\hline $\mathbf{0 . 0}$ & $9.697 \times 10^{-10}$ & $7.854 \times 10^{-8}$ \\
\hline 0.2 & $2.710 \times 10^{-10}$ & $4.413 \times 10^{-5}$ \\
\hline 0.4 & $8.833 \times 10^{-11}$ & $1267 \times 10^{-5}$ \\
\hline 0.6 & $2.048 \times 10^{-9}$ & $6.604 \times 10^{-5}$ \\
\hline 0.8 & $5.607 \times 10^{-9}$ & $1.609 \times 10^{-4}$ \\
\hline 1.0 & $1.077 \times 10^{-8}$ & $3.647 \times 10^{-4}$ \\
\hline
\end{tabular}

Table 3. Comparison of the absolute errors for Example 2

\begin{tabular}{|c|c|c|}
\hline $\mathbf{x}$ & $\operatorname{LSCBM}_{u_{1}(x)}$ & ADM [20] \\
\hline 0.0 & $9.031 \times 10^{-9}$ & $0.000 \times 10^{+10}$ \\
\hline 0.2 & $1.293 \times 10^{-7}$ & $3.381 \times 10^{-3}$ \\
\hline 0.4 & $2.469 \times 10^{-7}$ & $6.510 \times 10^{-3}$ \\
\hline 0.6 & $3.036 \times 10^{-7}$ & $9.942 \times 10^{-3}$ \\
\hline 0.8 & $2.411 \times 10^{-7}$ & $1.372 \times 10^{-2}$ \\
\hline 1.0 & $1.031 \times 10^{-9}$ & $1.786 \times 10^{-2}$ \\
\hline
\end{tabular}

Table 4. Comparison of the absolute errors for Example 2

\begin{tabular}{|l|c|c|}
\hline $\mathrm{x}$ & $\mathrm{LSCBM} u_{2}(\boldsymbol{x})$ & $\mathrm{ADM}[20]$ \\
\hline $\mathbf{0 . 0}$ & $\mathbf{0 . 0 0 0} \times 10^{+10}$ & $\mathbf{0 . 0 0 0} \times 10^{+10}$ \\
\hline 0.2 & $6.632 \times 10^{-9}$ & $4.753 \times 10^{-4}$ \\
\hline 0.4 & $1.274 \times 10^{-8}$ & $1.130 \times 10^{-3}$ \\
\hline 0.6 & $1.562 \times 10^{-8}$ & $1.876 \times 10^{-3}$ \\
\hline 0.8 & $1.261 \times 10^{-8}$ & $2.689 \times 10^{-3}$ \\
\hline 1.0 & $1.000 \times 10^{-9}$ & $3.534 \times 10^{-3}$ \\
\hline
\end{tabular}

Table 5. Comparison of the absolute errors for Example 3

\begin{tabular}{|l|c|c|}
\hline $\mathrm{x}$ & $\mathrm{LSCBM} \mathrm{u_{1 } ( x )}$ & $\mathrm{ADM}[20]$ \\
\hline $\mathbf{0 . 0}$ & $\mathbf{0 . 0 0 0} \times \mathbf{1 0}^{+10}$ & $\mathbf{0 . 0 0 0} \times \mathbf{1 0}^{+10}$ \\
\hline 0.2 & $2.624 \times 10^{-10}$ & $6.852 \times 10^{-4}$ \\
\hline 0.4 & $7.872 \times 10^{-10}$ & $2.386 \times 10^{-3}$ \\
\hline 0.6 & $1.181 \times 10^{-9}$ & $4.950 \times 10^{-3}$ \\
\hline 0.8 & $1.050 \times 10^{-9}$ & $8.309 \times 10^{-3}$ \\
\hline 1.0 & $\mathbf{0 . 0 0 0} \times \mathbf{1 0}^{+10}$ & $1.241 \times 10^{-2}$ \\
\hline
\end{tabular}

Table 6. Comparison of the absolute errors for Example 3

\begin{tabular}{|l|c|c|}
\hline $\mathrm{x}$ & $\mathrm{LSCBM} \mathrm{u_{2 } ( x )}$ & $\mathrm{ADM}[20]$ \\
\hline $\mathbf{0 . 0}$ & $\mathbf{0 . 0 0 0} \times \mathbf{1 0}^{+10}$ & $\mathbf{0 . 0 0 0} \times 10^{+10}$ \\
\hline 0.2 & $3.546 \times 10^{-8}$ & $2.400 \times 10^{-3}$ \\
\hline 0.4 & $1.321 \times 10^{-7}$ & $3.307 \times 10^{-3}$ \\
\hline 0.6 & $2.755 \times 10^{-7}$ & $5.331 \times 10^{-3}$ \\
\hline 0.8 & $4.509 \times 10^{-7}$ & $7.662 \times 10^{-3}$ \\
\hline 1.0 & $6.440 \times 10^{-7}$ & $1.029 \times 10^{-2}$ \\
\hline
\end{tabular}

\section{CONCLUSION}

In this study, Bernstein polynomials, least square collocation together with Caputo properties are used to find the solution of system of linear fractional integro- differential equations. There is a high rate of convergence of the approximate solutions to the exact solutions. Specifically, the performance of the proposed method was compared with existing results in the literature and are found to be more efficient in the terms of accuracy. It is also observed that the number of iterations needed in solving the problems using the proposed method is few and with lower values of $M$ (the degree of the approximant). 
Acknowledgement: Authors thank those who contributed to write this article and give some valuable comments.

Funding Statement: The authors received no specific funding for this study.

Conflicts of Interest: The authors declare that they have no conflicts of interest to report regarding the present study.

\section{REFERENCES}

[1] Adam, L., "Fractional Calculus: history, Definition and application for the engineer," Department of Aerospace and Mechanical Engineering University of Notre Dame, IN 46556, U.S.A. (2004)

[2] Awawdeh, F., Rawashdeh, E. A. and Jaradat, H. M., "Analytic solution of fractional integro-differential equations," Annals of the University of CraiovaMathematics and Computer Science Series (2011), vol. 38 , no. 1, pp. 1-10.

[3] Caputo, M., "Linear models of dissipation whose Q is almost frequency Independent," Geophysical Journal International (1967), vol. 13, no. 5, pp.529 - 539.

[4] Dr.Osama H. Mohammed and Sarmad A. Altaie, "Approximate solution of Fractional Integro-Differential Equations by using Bernstein polynomials, Engineering and Technology Journal (2012), vol. 30 no. 8, pp. 13621373.

[5] Aysegul, D. and Dilek V. B., "Solving fractional Fredholm integro-differential equations by Laguerre polynomials", Sains Malaysiana (2019),Vol. 48, no. 1, pp. $251-257$

[6] Dilkel,V. B. and Aysegül, D.,"Applied collocation method using Laguerre polynomials as the basis functions," Advances in difference equations a springer open journal (2018), pp. 1- 11 .

[7] Ma, X. and Huang, C., "Spectral collocation method for linear fractional integro- differential equations," Applied Mathematical Modelling (2014), vol. 38, no. 4, pp. 14341448.

[8] Ma, X, and Huang, C "Numerical solution of fractional integro-differential equations by a hybrid collocation method," Applied Mathematics and Computation (2013), vol. 219, no. 12, pp. 6750-6760.

[9] Amr, Y. A., Mahdy, M. S. and Youssef, E. S. M., "Solving fractional integro-differential equations by using Sumudu transform method and Hermite spectral collocation method," Computers, Materials and Continua (2018), vol. 54, no. 2, pp. 161-180.

[10] Alkan, S. and Hatipoglu, V. F., "Approximate solutions of Volterra-Fredholm integro-differential equations of fractional order," Tbilisi Mathematical Journal (2017), vol. 10, no. 2, pp.1-13

[11] Mahdy A. M. S. and Mohamed, R. M. H.,"Numerical studies for solving system of linear fractional integrodifferential equations by using least squares method and shifted Chebyshev polynomials," Journal of Abstract and Computational Mathematics (2016), vol. 1, no. 24, pp. 24-32,2016

[12] Mohammed, D. Sh., "Numerical solution of fractional integro-differential equations by least squares method and shifted Chebyshev polynomial," Mathematical Problems in Engineering (2014), Article ID 431965, no. 1, pp. 1-5.

[13] Mohammed, D. Sh., "Numerical solution of fractional singular integro-differential equations by using Taylor series expansion and Galerkin method," Journal of Pure and Applied Mathematics: Advances and Applications (2014), vol. 12 no. 2, pp. 129-143.

[14] Nemati, S., Sedaghatb, S. and Mohammadi, I., "A fast numerical algorithm based on the second kind Chebyshev polynomials for fractional integro-differential equations with weakly singular kernels,",Journal of Computational and Applied Mathematics (2016), vol. 308 , pp. 231-242

[15] Yi, M. and Huang, J., "CAS wavelet method for solving the fractional integro-differential equation with a weakly singular kernel," International Journal of Computer Mathematics (2015), vol. 92, no. 8, pp. 1715-1728.

[16] Setia, A., Liu, Y. and A. S. Vatsala, A. S."Numerical solution of Fredholm-Volterra Fractional integrodifferential equation with nonlocal boundary conditions," Journal of Fractional Calculus and Applications (2014), vol. 5, no. 2, pp. 155-165.

[17] Oyedepo, T., Taiwo, O. A., Adebisi, A. F., Raji, M. T., Ajisope M. O. et al, "Bernstein modified Homotopy perturbation method for the solution of Volterra Fractional integro-differential equations," Pasifi Journal of Science and Technology (2021), vol. 22, no. 1, pp., 30-36.

[18] C. Edwards, "Math 312 Fractional calculus final presentation,"[video] Available at: https://www.youtube.com/watch?v=CsJa3XiOmfs [Accessed 20 Sep. 2018].

[19] T. Grant, (2014) Math 336 Approximating Continuous Functions and Curves using Bernstein Polynomials.

[20] Saleh, M. H., Mohamed, S. H., Ahmed, M. H. and Marjan, M. K., "System of linear fractional integrodifferential equations by using Adomian decomposition method," International Journal of Computer Applications (2015), vol. 121, no. 24, pp., 9 - 19. 